\title{
An Algebraic Method for Solving a Class of Indefinite Integrals
}

\author{
Fucheng Liao ${ }^{1, a}$, Xiao Wang ${ }^{2, b}$, Xuejin Yan ${ }^{3, c}$ and Di Wang ${ }^{4, d}$ \\ School of Mathematics and Physics, University of Science and Technology Beijing, Beijing 100083, \\ China, \\ afcliao@ustb.edu.cn, b1928903845@qq.com, 'cyanxuejin1220@163.com, 'wangdi_1982@163.com
}

Keywords: Indefinite integral, Linear space, Derivative operator, Basis function, Inverse transformation

\begin{abstract}
In this paper, an algebraic method for solving indefinite integrals of a class of functions is proposed. The essence of the method is to construct a linear space spanned by the class of functions; thus, the derivative operator is a linear transformation of the linear space. By calculating the value of the derivative operator on the basis function, the matrix of the derivative operator is obtained, and the inverse matrix is derived: this is the matrix of the inverse transformation for the derivative operator. If a function is expressed as a linear combination on the basis of the constructed linear space, one of its primitive functions can be simply represented by using the above inverse transformation. Finally, the indefinite integral of the function is obtained by adding an integral constant to the primitive function. The salient feature of this paper is to avoid the relatively complex integral calculation.
\end{abstract}

\section{Introduction}

The method for solving indefinite integrals of continuous functions is the basic operation in mathematical analysis [1]. Indefinite integral calculation has been extensively discussed in mathematical analysis textbooks. In addition, a great deal of research on how to solve indefinite integrals can be found in vast arrays of academic and teaching research journals [2,3]. Nevertheless, most of these papers categorize and summarize existing integral methods. Ref. [4] studied the solution of indefinite integrals by inverse matrix, but its narrative is difficult to understand.

In fact, the theories of advanced algebra [5] and mathematical analysis are deeply related. The core idea of this paper is to study the indefinite integrals of a class of derivative functions, via sufficient utilization of the linear transformation in advanced algebra. According to the problem requirements, we construct a linear space containing the integrand, and then the derivative operator is the linear transformation of this linear space. The action of inverse transformation on a function is one of its primitive functions, so the indefinite integral of the function is derived by adding an arbitrary constant. The result of Ref. [4] is a corollary of this paper.

\section{Algebraic method to solve $\int x^{2} e^{x} d x$}

As we know, in a certain sense, an integral is the inverse operation of a derivative. In order to solve the indefinite integral of $f(x)=x^{2} e^{x}$, we first calculate its derivative. Constructing an operator as

$$
D=\frac{d}{d x}
$$

Then $D(f)=\frac{d f}{d x}$, and we have

$$
D\left(x^{2} e^{x}\right)=x^{2} e^{x}+2 x e^{x}
$$

We note that $x e^{x}$ appears on the right side of the equation, and its derivative can be processed as

$D\left(x e^{x}\right)=x e^{x}+e^{x}$

Similarly, we have

$D\left(e^{x}\right)=e^{x}$ 
From (1), (2), and (3), we derive

$$
\left\{\begin{array}{c}
D\left(x^{2} e^{x}\right)=x^{2} e^{x}+2 x e^{x}+0 e^{x} \\
D\left(x e^{x}\right)=0 x^{2} e^{x}+x e^{x}+e^{x} \\
D\left(e^{x}\right)=0 x^{2} e^{x}+0 x e^{x}+e^{x}
\end{array}\right.
$$

Namely,

$$
\left(D\left(x^{2} e^{x}\right), D\left(x e^{x}\right), D\left(e^{x}\right)\right)=\left(x^{2} e^{x}, x e^{x}, e^{x}\right)\left(\begin{array}{ccc}
1 & 0 & 0 \\
2 & 1 & 0 \\
0 & 1 & 1
\end{array}\right)
$$

Therefore, by taking three linearly independent functions as $x^{2} e^{x}, x e^{x}, e^{x}$, and choosing ordinary vector addition and scalar multiplication of the functions, a three-dimensional linear space can be spanned as

$$
S=L\left(x^{2} e^{x}, x e^{x}, e^{x}\right)
$$

Where $x^{2} e^{x}, x e^{x}, e^{x}$ is a basis of $S$. We can prove that $D$ is a linear transformation of $S$; then the matrix representation of $D$ under the basis $x^{2} e^{x}, x e^{x}, e^{x}$ is that:

$$
A=\left(\begin{array}{lll}
1 & 0 & 0 \\
2 & 1 & 0 \\
0 & 1 & 1
\end{array}\right)
$$

From $|A|=1 \neq 0$, we know that $A$ is invertible, and the inverse matrix of $A$ can be derived

$$
A^{-1}=\left(\begin{array}{ccc}
1 & 0 & 0 \\
-2 & 1 & 0 \\
2 & -1 & 1
\end{array}\right)
$$

This implies that the corresponding linear transformation $D$ is invertible, and we get

$\left(D^{-1}\left(x^{2} e^{x}\right), D^{-1}\left(x e^{x}\right), D^{-1}\left(e^{x}\right)\right)=\left(x^{2} e^{x}, x e^{x}, e^{x}\right) A^{-1}$

Namely,

$$
\left\{\begin{array}{l}
D^{-1}\left(x^{2} e^{x}\right)=x^{2} e^{x}-2 x e^{x}+2 e^{x} \\
D^{-1}\left(x e^{x}\right)=x e^{x}-e^{x} \\
D^{-1}\left(e^{x}\right)=e^{x}
\end{array}\right.
$$

$D^{-1}(f(x))$ represents one of the primitive functions of $f(x)$, and satisfies

$$
\left(D^{-1} D\right)(f(x))=\left(D D^{-1}\right)(f(x))=f(x)
$$

Therefore, the indefinite integral of $f(x)$ can be expressed as

$$
\int f(x) d x=D^{-1}(f(x)+C
$$

So, from the first equation of (1), we obtain

$$
\int x^{2} e^{x} d x=x^{2} e^{x}-2 x e^{x}+2 e^{x}+C
$$

This method of solving the indefinite integral is suitable for a large class of functions. Note that we don't really calculate the integral itself, so the problem is simplified.

\section{Algebraic method to solve $\int x^{n} e^{x} d x$ (where $n$ is a positive integer)}

Based on the above discussion, we know that if we want to calculate the indefinite integral of a function by using the method of solving the inverse transformation, the key is to find a class of functions such that derivatives of the functions also belong to it. Therefore, we can construct a suitable linear space and linear transformation. 
Note that the derivative of the function $x^{n} e^{x}$ can be expressed as a linear combination of the same form function $x^{k} e^{x}(k \leq n)$, so the results of the previous section can be extended to solve the indefinite integral of $f(x)=x^{n} e^{x}$.

We construct a linear space as

$$
S=L\left(x^{n} e^{x}, x^{n-1} e^{x}, \cdots, x e^{x}, e^{x}\right)
$$

Note that $x^{n} e^{x}, x^{n-1} e^{x}, \cdots, x e^{x}, e^{x}$ is linearly independent, so it is a basis of $S$. The linear transformation on $S$ is defined as

$$
D=\frac{d}{d x}
$$

By calculating the derivatives of $x^{n} e^{x}, x^{n-1} e^{x}, \cdots, x e^{x}, e^{x}$ respectively, we get

$$
\left(D\left(x^{n} e^{x}\right), D\left(x^{n-1} e^{x}\right), \cdots, D\left(x e^{x}\right), D\left(e^{x}\right)\right)=\left(x^{n} e^{x}, x^{n-1} e^{x}, \cdots, x e^{x}, e^{x}\right) A,
$$

where the matrix representation of $D$ on the basis of $x^{n} e^{x}, x^{n-1} e^{x}, \cdots, x e^{x}, e^{x}$ is that

$$
A=\left(\begin{array}{ccccccc}
1 & 0 & 0 & \ldots & 0 & 0 & 0 \\
n & 1 & 0 & \ldots & 0 & 0 & 0 \\
0 & n-1 & 1 & \ldots & 0 & 0 & 0 \\
\ldots & \ldots & \ldots & \ldots & \ldots & \ldots & \ldots \\
0 & 0 & 0 & \ldots & 1 & 0 & 0 \\
0 & 0 & 0 & \ldots & 2 & 1 & 0 \\
0 & 0 & 0 & \ldots & 0 & 1 & 1
\end{array}\right)
$$

It is easy to see that $A$ is invertible. We know that $A^{-1}$ is the matrix representation of $D^{-1}$ on the basis of $x^{n} e^{x}, x^{n-1} e^{x}, \cdots, x e^{x}, e^{x}$, and the first column of $A^{-1}$ is the coordinate of $D^{-1}\left(x^{n} e^{x}\right)$. If we want to calculate the indefinite integral of $x^{n} e^{x}$, we only need to obtain the first column of $A^{-1}$. The column vector consisting of the first column of $A^{-1}$ is the solution of the following linear equation $A y=b$,

where $b=(1,0, \cdots, 0)^{T}$. So, we take the elementary transformation of rows for the matrix $(A, b)$; when $A$ has been transformed to an identity matrix, the block of $b$ is transformed into the first column of $A^{-1}$. Based on the form of $A$, it is easy to see that

$$
A^{-1} b=\left(1,-n, n(n-1), \cdots,(-1)^{n-2} \frac{n !}{2 !},(-1)^{n-1} n !,(-1)^{n} n !\right)^{T}
$$

Thus, we get

$$
D^{-1}\left(x^{n} e^{x}\right)=\left(x^{n} e^{x}, x^{n-1} e^{x}, \cdots, x e^{x}, e^{x}\right) A^{-1} b=e^{x}\left[x^{n}-n x^{n-1}+n(n-1) x^{n-2}-\ldots+(-1)^{n} n !\right]
$$

Adding the integral constant, we have

$$
\int x^{n} e^{x} d x=e^{x}\left[x^{n}-n x^{n-1}+n(n-1) x^{n-2}-\cdots+(-1)^{n} n !\right]+C
$$

\section{Algebraic method to solve indefinite integrals}

The above-mentioned method can be extended. We assume that functions $f_{1}(x), f_{2}(x), \cdots, f_{n}(x)$ are linearly independent, and their derivatives can also be expressed as linear combinations of them. Then the previous method can be used to solve the indefinite integral of the functions, as follows:

$1)$ : Constructing a linear space spanned by the functions $f_{1}(x), f_{2}(x), \cdots, f_{n}(x)$ as

$$
\mathrm{S}=\mathrm{L}\left(f_{1}(x), f_{2}(x), \cdots, f_{n}(x)\right)
$$

Note that $f_{1}(x), f_{2}(x), \cdots, f_{n}(x)$ is a basis of $S$. 
2): The derivative operator $D=\frac{d}{d x}$ is a linear transformation of the linear space, so by taking the following derivatives of $f_{1}(x), f_{2}(x), \cdots, f_{n}(x)$ :

$$
\left(D\left(f_{1}(x)\right), D\left(f_{2}(x)\right), \cdots, D\left(f_{n}(x)\right)\right)=\left(f_{1}(x), f_{2}(x), \cdots, f_{n}(x)\right) A
$$

the matrix $A$ representation of $D$ is obtained.

$3)$ : Calculating $A^{-1}$. Since it is a representation of $D$, we get

$$
\left(D^{-1}\left(f_{1}(x)\right), D^{-1}\left(f_{2}(x)\right), \cdots, D^{-1}\left(f_{n}(x)\right)\right)=\left(f_{1}(x), f_{2}(x), \cdots, f_{n}(x)\right) A^{-1}
$$

4): Solving the desired indefinite integral. Let $A^{-1}=\left(\begin{array}{llll}b_{1} & b_{2} & \cdots & b_{n}\end{array}\right)$. For example, if we want to solve the indefinite integral of a function $f(x) \in S$, we first take $f(x)$ expressed as linear combination of $f_{1}(x), f_{2}(x), \cdots, f_{n}(x)$ :

$$
f(x)=k_{1} f_{1}(x)+k_{2} f_{2}(x)+\cdots+k_{n} f_{n}(x)=\left(f_{1}(x), f_{2}(x), \cdots, f_{n}(x)\right)\left(\begin{array}{c}
k_{1} \\
k_{2} \\
\vdots \\
k_{n}
\end{array}\right)
$$

Then, we have

$$
\begin{aligned}
\int f(x) d x & =D^{-1} f(x)+C=D^{-1}\left(f_{1}(x), f_{2}(x), \cdots, f_{n}(x)\right)\left(\begin{array}{c}
k_{1} \\
k_{2} \\
\vdots \\
k_{n}
\end{array}\right)+C \\
& =\left(f_{1}(x), f_{2}(x), \cdots, f_{n}(x)\right) A^{-1}\left(\begin{array}{c}
k_{1} \\
k_{2} \\
\vdots \\
k_{n}
\end{array}\right)+C
\end{aligned}
$$

where $C$ is an integral constant. Thus, the problem of the indefinite integral has been transformed into the expression through the basis of $S$ in the form of a linear combination.

\section{Conclusions}

The algebraic method for solving the indefinite integrals of a class of functions is investigated in this paper. The salient feature of the method is that direct calculation of the integral is avoided. As long as the derivatives of a class of functions still belong to the class of functions, we can solve the indefinite integral by the method offered in this paper. In addition, the method proposed here can extend to solve a class of ordinary differential equations and higher-order ordinary differential equations.

\section{Acknowledgements}

This work was financially supported by the National Natural Science Foundation of China (61174209), the Oriented Award Foundation for Science and Technological Innovation, Inner Mongolia Autonomous Region, China (2012), Project of research teaching of demonstration lessons at the University of Science and Technology Beijing, 2011 (JY2011SFK10), and Building Project of personnel training, "Research and Practice of relying on the advantages of discipline to train top creative talents, Beijing (2012)". 


\section{References}

[1] Y.L. Liu, P.R. Fu and D. Lin: Mathematical Analysis, Higher Education Press, 5th Edition, BJ (2008)

[2] D.S. Yu, Y.Z. Yu: Studies in College Mathematics, Vol. 11 (2008), p. 35

[3] Y.P. Huang: Journal of Hunan University of Science and Engineering, Vol. 31 (2010), p. 28

[4] L.H. Wang, F.R. Wang: Journal of Henan Institute of Education (Natural Science Edition), Vol. 20 (2011), p. 1

[5] E.F. Wang, S.M. Shi: Advanced Algebra, Higher Education Press, 3rd Edition, BJ (2003) 